\title{
L'enseñu virtual de la llingua asturiana (1996-2019): averamientu etnográficu, estudiu evaluativu y propuestes de futuru / Asturian language e-learning (1996-2019): ethnographic approach, evaluative study and future purposes
}

\author{
CARLOS SUARI RODRIGUE \\ UNIVERSITAT ROVIRA I VIRGILI \\ ORCID 0000-0002-0575-988X
}

\begin{abstract}
RESUME: A mediaos de los años 1990, cola xeneralización d'internet, dalgunes comunidaes falantes de llingües minoritaries vieron apaecer nueves y bones opciones pa caltener y enanchar los sos usos. Remanando les ferramientes corrientes de la rede o tresnando otres específicamente didáctiques, l'alfabetización per medios telemáticos convirtióse nuna de les grandes oportunidaes que-yos apurría a estos idiomes la entrada na era dixital. Nel casu del asturianu, aparte de dalgunes propuestes parciales, el dominiu de la formación en llinia ta y tuvo protagonizáu por cuatro programes d'enseñu virtual (PEVA). Col oxetivu de facer un retratu de conxuntu del qu'estrayer propuestes pal futuru, esta investigación enfotóse n'analizar la constitución y los procesos de desenvolvimientu de los PEVA, los sos componentes, los sos resultaos educativos y los sos efectos na normalización social del idioma. De primeres, un percorríu pela bibliografía global y particular d'esti dominiu dibuxa un marcu teóricoconceptual que fala de grandes potenciales y desafíos metodolóxicos au asitiar el casu de la llingua asturiana, qu'inda nun xenerare investigación nenguna hasta güei. Darréu, cola esposición del diseñu metodolóxicu, amosaráse'l calter mestu de la investigación na que s'enconta esti testu, una busca d'información qu'amiesta ferramientes cualitatives y cuantitatives como la observación, la entrevista y el cuestionariu. Too siguío, en dos apartaos dixebraos pero consecutivos, asoléyase una esbilla de los resultaos collechaos: un averamientu etnográficu basáu nos datos empíricos y un estudiu evaluativu fundamentáu estadísticamente. Xuntando les dos llinies metodolóxiques de la investigación nuna síntesis final, conoceráse por qué y cómo se desenvolvió'l campu del enseñu virtual del asturianu nesti cuartu de sieglu postreru, los sos efectos positivos na normalización llingüística y los resultaos desiguales de los PEVA na so misión pedagóxica. Peracabando l'artículu, dándo-yos traza de propuestes, formúlense en positivo una riestra de conclusiones, les más sólides de la investigación, aguardando qu'esti tipu de programes xorrezan nel futuru sobre la reflexón de la esperiencia.
\end{abstract}

Pallabres clave: llingua minoritaria, alfabetización, normalización, pedagoxía, internet, etnografía.

ABSTRACT: In the mid 1990s, with the general widespread of the Internet, some communities of speakers of minority languages took advantages of the new options to 
preserve and enlarge its uses. Employing the tools offered by the Internet or exploiting other didactic instruments, multimedia literacy became one of the best opportunities to introduce these languages into the digital era. As regards, the Asturian language, besides some partial proposals, online training programs have been characterized by four online learning platforms (PEVA). Aiming at providing a general overview in order to make proposals for the future, in this study I have explored the constitution and the development of PEVA, their main components, their results from an educational point of view and their effects on the social normalization process of the language. Firstly, I will present some of the general and particular bibliography about this topic to outline a theoretical and conceptual framework, which may include potential achievements and methodological challenges for the Asturian case. Secondly, as the methodological design is exposed, a mixed nature for the research will be proposed, involving a quest for information with both qualitative and quantitative tools such as observation, interviews and questionnaires. Then, a sample of the results will be described: an ethnographic approach based on empirical data and a statistically-based evaluative approach. The two methodological lines will be brought together into a final synthesis in order to illustrate why and how the field of online virtual learning evolved in the referred period, its positive impact on normalization and the unequal results of the so-called PEVA from a pedagogical point of view. To end with, some positive conclusions, expecting this kind of educational programs to be improved, will be drawn.

Key words: minority language, literacy, normalization, pedagogy, Internet, ethnography.

\section{INTRODUCCIÓN}

Una güeyada xeneral al traviés d'internet val pa ver que los idiomes mayoritarios tienen güei una bona cuota dientro de la ufierta de formaciones en llinia, tando ente lo más demandao pola ciudadanía y esfrutando, como «grandes llingües», d'una posición d'importancia económica y prestixu social que les coloca como ún de los motores de la industria del enseñu virtual. Otres llingües falaes por comunidaes más pequeñes, ensin l'interés comercial d'aquelles otres y de xuru que d'un xeitu mui diferente, tienen tamién el so espaciu na rede (Assini 2014: 28). Y ye que, como se va ver darréu, munches d'estes llingües, minoritaries y en riesgu de desaniciu, ente les que ta l'asturianu (Moseley 2010), yá foron quien a llevantar los sos propios programes formativos n'internet. Sicasí, la so probitú de medios fai que'l desenvolvimientu estratéxicu y la caltenibilidá d'estos proyectos protagonizaos por llingües «pequeñes» nun algamen la estabilidá afayadiza (Hugo 2015). Entós, si l'apaición d'estos idiomes nel tarrén pedagóxico-virtual nun vien del porgüeyu mercantil, ¿cuála ye la so razón motora? ¿Quién encabeza estes iniciatives? ¿En qué s’enfoten les persones que les echen a andar? 
Pal casu específicu asturianu, gracies a una primer observación superficial, pudo sabese que son cuatro les plataformes, programes $-\mathrm{o}$ cursos, en resumíes cuentes- que güei ufren la posibilidá de deprender esti idioma n'internet y que d'equí p'alantre van apaecer conseñaos cola sigla PEVA (programes d'enseñu virtual del asturianu). Pela mesma vía de comprobación, nun abulta qu'esistieren nin esistan otres propuestes asemeyaes -anque sí nicios o elementos parciales-. Nuna repasada a les fontes bibliográfiques, nun aprució analís nengún d'esos cuatro PEVA. Atopáronse namás delles reflexones xenerales alredor de les posibilidaes y retos que lo dixital-y traxo al asturianu (García \& Sánchez 2008, Morán 1995, Núñez 2000), estudios concretos sobre l'usu de les tecnoloxíes «nueves» nes aules reglaes de llingua asturiana (Berros 2007 y 2008, Guardado 2002, Ovide 2005) o, más a lo llargo, sobre otres formes d'aplicación allugaes nel rodiu de la llingua asturiana y les TIC (Galán 2014, González 2014, González-Riaño et al. 2010, Iglesias \& Yeguas 2014, Saurí 2004).

En desllindando'l marcu teóricu global -y n'asitiando nelli l'ámbitu llingüísticu asturianu- van presentase la metodoloxía, un averamientu etnográficu y un estudiu evaluativu, colos qu'algamar, yá d'últimes, delles conclusiones espresaes como propuestes de futuru.

\section{ANTECEDENTES Y RODIADA DE LA INVESTIGACIÓN}

Na bibliografía qu'enconta esta revisión atópense referencies, dende enfoques mui estremaos, a unes 70 llingües. Ente ello hai llibros y artículos qu'apurren reflexones sobre l'impactu xeneral de les tecnoloxíes virtuales nes llingües minoritaries, hai obres centraes nes particularidaes del so enseñu y hailes, p'acabu, que xunten dambos aspectos. D'otru llau, afayáronse referencies que recueyen esperiencies d'enseñu virtual d'estos idiomes: delles parciales, otres al traviés de programes integrales y otres más, au se recoyó'l surdimientu de metodoloxíes específiques pa esti tipu de formaciones. Rematando, tamién se revisaron delles obres sociollingüístiques y otres dedicaes al estudiu tresversal del espoxigue d'internet.

\subsection{Bayura llingüistica y minoríes dixitalizaes}

La dificultá de definir, pola so naturaleza multifactorial, qué ye una llingua en riesgu de desaniciu apuerta incluso a ún de los principales catálogos d'esta mena d'idiomes (Moseley 2010). Sicasí, apúntense nelli dos dimensiones pa pescudar el peligru o la fraxilidá llingüística: el númberu de falantes que s'identifiquen cola llingua y el númberu y el tipu d'usos y 
funciones que tien. D'esta miente, l'amenaza de desaniciu pue venir de la mengua na cantidá de persones qu'entienden l'idioma como propiu, pero tamién de que se reduzan los sos usos, dexando de tresmitilu a les xeneraciones vinientes y perdiendo adulces la so funcionalidá (Simons \& Fennig 2018). Anguaño, el mundu tien 7.097 llingües falaes por 6.78 millones de persones, pero la so distribución nun ye proporcional: el $94.4 \%$ d'elles correspuende al $5.6 \%$ de la población. Anque la media de falantes/llingua ye de 955.000 , hai un $80.7 \%$ d'idiomes que nun algama siquiera los 100.000 falantes (cálculos iguaos colos datos de Simons \& Fennig 2018).

Fai falta esclariar otros dos conceptos frecuentes nesti ámbitu: llingua minoritaria -la que fala un grupu humanu pequeñu dientro d'un conxuntu poblacional mayor- y llingua minorizada - la que ta restrinxida nos sos ámbitos y funciones per aciu d'un procesu políticu de subordinación llingüística- (Termcat 2018). Fecha la xebra semántica, cuídase que más que sinónimos, estos términos coinciden davezu en contestu al tar, dalgunes llingües, en toes tres condiciones: minoritaries (pocos falantes), minorizaes (arrequexamientu) y amenazaes (poles poques opciones de sobrevivivir).

\section{Llingües minoritaries y enseñu virtual}

Andaba l'añu 1996 cuando'l mundu crució la llende del 1\% de población usuario d'internet -la Xunión Europea pasare un añu primero-. Sicasí, los Estaos Xuníos, Finlandia, Suiza, Australia y Suecia yá lo fixeren en 1991 (World 2018). Pela cueta, los 7 estaos subdesendolcaos o en víes de desendolcu que concentren la metá de les llingües minoritaries del planeta (Moseley 2018) entá teníen, naquel añu 1996, unes tases d'entrada a la rede perbaxes: el $0.0 \%$ en Papúa y Nueva Guinea, La China, El Camerún y La India; $0.1 \%$ n'Indonesia y Nixeria; y el $0.2 \%$ en Méxicu. Tovía diben entardar ente 4 y 9 años n'algamar el 1\% d'usuarios d'internet. Trancando la primer década del sieglu XXI (2009), un 25\% de la población mundial usaba internet y La China y Méxicu yá taben nesti mesmu datu. La India, pel so llau, llegaba a esti puntu nel 2015 y Nixeria, Indonesia y El Camerún, un añu depués, cuando la media mundial yá taba nel $46 \%$ (estudiu comparativu encontáu en World 2018). En 2018, la entrada media mundial n'internet yera del 55\% (Kemp 2018), pero Papúa y Nueva Guinea nun algamaben siquieramente'l 20\% (World 2018). Esti resume del inxerimientu del mundu n'internet, y viceversa, desplica que la llegada de les primeres reflexones y compilatorios d'esperiencies sobre l'enseñu virtual de les llingües minoritaries s'allargare ente mediaos de la década de 1990 y l'actualidá (dellos idiomes «pequeños» tán n'estaos desendolcaos, pero otros non); y xustifica lo que yá dixeren Cunliffe \& Herring (2005): estos idiomes tán na rede con un programariu davezu anticuáu y que dacuando ta vehicularizáu per aciu de llingües 
mayoritaries, cosa que nun fai otro que recalcar la so rellación de subordinación.

\section{Internet, una rede que promueve la normalización llingüística}

Dende les primeres esperances trayíes pola virtualidá a les llingües minoritaries (Ostler 2001), la bibliografía vien discutiendo les potencialidaes normalizadores de la dixitalización de les minoríes llingüístiques. Asina, sorrayóse la so capacidá d'axuntar comunidaes, davezu espardíes, aumentando les conexones interpersonales y, poro, l'enfotu en que sobrevivan les propies llingües amenazaes (Evas 2014). Destacóse tamién que dalgunes d'estes comunidaes de falantes, dientro del escenariu dixital, lleguen a tar más valoraes que presencialmente (Hogan-Brun 2011). Coles mesmes, constatóse que les llingües minoritaries yá tán arriqueciéndose gracies al so usu con ferramienta virtual (McAllister et al. 2013).

Un estudiu protagonizáu por 9 idiomes minoritarios europeos comprobó que los más d'ellos tán presentes n’internet pente medies de páxines web y redes sociales y qu'inclusive los medios de comunicación públicos y mayoritarios tienen espacios nestos idiomes (Ferré-Pavia et al. 2018). Anque tán probaos el crecimientu d'estes llingües na rede y los beneficios sociollingüísticos d'ello, n’especial ente la mocedá (Moriarty 2011), tamién ta recoyida la necesidá de revisar los averamientos, davezu intuitivos y espontáneos, cola idea d'ameyorar la coordinación y facelos más estratéxicos (Hogan-Brun 2011: 328).

Tiense argumentao que la propia introducción de les minoríes llingüístiques na rede espándeles funcionalmente, aumenta la so visibilidá social (Cormack 2005), enancha'l repertoriu d'usos posibles, ameyora'l so prestixu n'asociación a la idea de modernidá $y$, en resume, failes más atrayibles (Eisenlohr 2004). Sobre'l manexu planificáu d'internet n'estratexes de normalización social, espunxéronse tamién qué factores indiquen l'ésitu nos estudios de Cunliffe \& Herring (2005) y de Paricio \& Martínez (2010). Comparando llingües mayoritaries y minoritaries, observóse que les «pequeñes» paecen más abiertes y granibles na xeneración d'usos nuevos por cuenta de movese nel contestu dixital (Gruffyd 2013).

\section{Enseñar llingües minoritaries nun ye cualquier enseñu}

Arnau (2005: 214) reparó nes fondes diferencies ente los enfoques y les práctiques docentes nes llingües minoritaries y mayoritaries, afitando que l'enseñu de la llingua minoritaria tien un conxutu de retos, dellos propios del enseñu de cualquier llingua y otros derivaos «de les sos condiciones de manifiesta inferioridá nel contestu social, nel espaciu y na valoración que se-y 
da». Coles mesmes, viose la necesidá d'arriquecer l'enseñu de la llingua minoritaria dándo-y procesos meyores que los de l'idioma mayoritariu: con actividaes interactivo-esperimentales, tecnoloxía y material diverso, usando la llingua fuera de los espacios formales (Arnau 2005: 215).

Anque la Xunión Europea vien ufriendo modelos de bones práctiques educatives pa los idiomes minoritarios más protexíos por llei (Van Dongera et al. 2017), nun hai qu'escaecer qu'en munchos otros casos l'alumnáu falante de llingües «pequeñes» nun atopa na escuela víes d'alfabetización -y sí, pela cueta, estremaes formes d'esclusión- (Dooly et al. 2009), riquiendo entós otru tipu de formación que, tando entamada pola so comunidá llinguiística o autónomamente, güei va pasar de xuru per internet.

Dalgunes de les ventayes de trabayar les llingües minoritaries con y al traviés de la rede son la so facilidá pa formar comunidaes educatives capaces a crear conteníos didácticos (Clark 2008) de manera constante (Cunliffe 2007) y la so naturaleza collaborativa y social (Mac Uidhilin 2013). Nun estudiu sobre 47 minoríes llingüístiques estremaes de dellos continentes indicóse que la tecnoloxía ufre grandes posibilidaes -positives nos más de los casos- pal so enseñu, con beneficios consistentes en toles comunidaes investigaes, con independencia del so tamañu y del so allugamientu xeográficu, rescamplando la necesidá d'adaptar la tecnoloxía a cada contestu específicu (Galla 2016). Tamién hai propuestes en cata d'unos principios xenerales d'intervención educativa y virtual sobre minoríes llingüístiques (Rau \& Meng-Chien 2007), na mesma llinia qu'otres (Dooly 2010) consideren estes práctiques como inherentemente complexes y anuncien el surdimientu de nueves metodoloxíes que s'afayen nes soluciones qu'internet ufierta a estos idiomes.

Por acabar d'amosar tola bayura d'enfoques, fai falta ver que dalgunes investigaciones recoyeron les esperiencies vivíes en proyectos específicos d'enseñu de llingües minoritaries calteníos pola alministración (Odé 2008, Edwards et al. 2002) y n'otros asemeyaos, xestionaos dende lo privao (Cunliffe et al. 2018, Jones 2015a, Villa 2002). D'otra miente, estudióse la combinación d'estes ferramientes específiques col usu de les redes sociales (Cunliffe et al. 2018) y dalgunes otres investigaciones centráronse nel enseñu d'esta mena d'idiomes basáu na participación nesos sitios tan comunes n'internet (Assini 2014, Clothey 2017, Cru 2015, De Graaf et al. 2015, Evas 2014, Johnson 2013, Jones 2015b, Reershemius 2017, Slimane 2008, Wagner 2013): delles, pa estrayer conclusiones bones d'aplicar en modelos metodolóxicos futuros, analizaron la naturaleza de les interacciones ente usuarios que s'alfabeticen nuna llingua minoritaria col encontu de les redes sociales (Reershemius 2017, Clothey 2017, Jones 2015b), mentes qu'Evas (2014) reflexonó alredor de los problemes surdíos a cuenta de la supremacía 
de los idiomes mayoritarios nestos puntos d'alcuentru virtual.

\subsection{El casu de la llingua asturiana}

L'asturianu ta catalogáu como amenazáu n'Ethnologue (Simons \& Fennig 2018) y nun grau de peligru de desaniciu definitivu y creciente na UNESCO - «l'idioma yá nun se depriende como llingua materna nel llar y los falantes más nuevos pertenecen a la xeneración paterna»- (Moseley 2010). Tien una comunidá falante de 110.000 persones como llingua principal y de 450.000 como secundaria (Simons \& Fennig 2018) y ta sometida a un procesu de perda d'usos y funcionalidá énte'l castellanu contra'l que se vienen proponiendo estremaes acciones de protección y normalización (García-Arias 1976, Xunta 1996, Academia 2018).

Garrando como referencia la comunidá autónoma asturiana, que ye au más falantes tien, obsérvase que la llegada de lo dixital nun tuvo especificidaes importantes en comparanza cola so rodiada (tolo más, una llixera anticipación) y sí una gran coincidencia cola media española a lo llargo'l tiempu, conque queda perriba de los niveles globales y un plizcu perbaxo de los estaos más tecnolóxicos (comparanza fecha cola información d'Asociación 2018, World 2018 y Kemp 2018).

\section{Oportunidaes y retos pal asturianu n'internet}

Tovía nun se midiere l'impactu de la rede n'Asturies (Asociación 2018) y yá se formularen delles reflexones sobre les oportunidaes que-y diba abrir al asturianu, pola so situación de minorización (Morán 1995). Nesta mesma llinia, manifestóse la necesidá de sofitar la creación de medios de comunicación dafechu n'asturianu, tando tamién ente ellos los encontaos nes tecnoloxíes «nueves» (Xunta 1996: 81).

Más alantre, volvieron a sorrayase la importancia y les dimensiones globales y cercanes de los proyectos industriales, sociales, culturales y políticos que se diben desenvolver nel nuevu mileniu cola llegada d'internet, reflexonando sobre les posibilidaes qu'ufríen nel casu asturianu, poles sos particularidaes culturales y pola condición social debilitada d'esta llingua (Núñez 2000: 71-84).

Atópense tamién na bibliografía otres referencies inespecífiques no que cinca a esta investigación, tocantes a ámbitos tan estremaos como los fenómenos neolóxicos rellacionaos cola tecnoloxía (Saurí 2004) o coles discusiones sociales y polítiques en plenu somorgamientu na virtualidá (García \& Sánchez 2008). 


\section{Sobre les esperiencies virtuales de la llingua asturiana}

Nel casu asturianu tamién hebo recoyida d'esperiencies y propuestes de recomendaciones práctiques de mui diferente xéneru depués de los primeros averamientos a internet de la comunidá llingüística. Dende l'estudiu y l'usu de ferramienta virtual concreta (González-Riaño et al. 2010), a la nueva terminoloxía del ámbitu tecnolóxico-dixital (Iglesias \& Yeguas 2014), pasando pelos peligros qu'acesmen a la mocedá na rede (González 2014). Estudiando los vezos de les persones de fala asturiana usuaries de la rede, concluyóse qu'estos representen una resistencia al dominiu de les grandes llingües asemeyada a la que se produz presencialmente, pero aprovechando lo que lo virtual ufierta a la continuidá na tresmisión del asturianu (Galán 2014: 210): la creación d'un espaciu au la llealtá llingüística de la comunidá ye quien a materializase y au la creciente emigración asturiana atopa un nexu dixital col so idioma maternu (Galán 2014: 219).

\section{Primeros reflexos dixitales nel enseñu del asturianu}

Cuasique la totalidá de reflexones y esperiencies atropaes al pasu pela bibliografía nel ámbitu del enseñu virtual del asturianu faen referencia a aportaciones dixitales ocasionales y auxiliares al contestu educativu regláu, específicamente, a l'asignatura de Llingua Asturiana que s'ufre n'institutos y escueles. En dellos casos, estúdiense les contribuciones xenerales de la informática y d'internet al enseñu escolar del asturianu (Guardado 2002, Ovide 2005, Berros 2008); n'otros, l'analís entra n'aplicaciones especializaes del mesmu contestu, como'l trabayu cola lliteratura infantil y xuvenil, per aciu de medios dixitales, dientro de la propia asignatura de Llingua Asturiana (Berros 2007).

Namás una contribución periodística recién recoyó la demanda social de programes de formación integrales acionaos pa l'alfabetización de persones adultes que, davezu, yá conocen y usen parcialmente l'asturianu, agora movíes pola sensibilidá énte'l so riesgu de desapaición (Sediles 2018).

\section{Metodoloxía}

Como yá s'espunxo acolumbrando esquemáticamente'l campu d'esta investigación, pescánciase qu'hai cuatro PEVA protagonizando les formaciones en llingua asturiana pela vía dixital, arriendes d'otres ferramientes sueltes, dalgunes d'elles yá ensin actividá. Sicasí, más alantre sedrá necesario xustificar si esta estremadura ente PEVA y otres propuestes tien una base empírica. Pel so llau, l'estudiu bibliográficu vien de poner enriba la mesa en 
qué llinies temátiques y con qué enfoques metodolóxicos ta investigándose na asturiana y n'otres comunidaes llinguiístiques minoritaries virtualizaes. Poro, conforme a Riba et al. (2011), lo qu'agora encartia ye formular el problema d'investigación y zarrar asina'l so nivel teórico-conceptual.

En ficies de caracterizar dafechu l'enseñu virtual del asturianu, diagnosticalu y, yá d'últimes, ufri-y delles propuestes de futuru, el trabayu de campu va dirixise a la formación de datos que dean consistencia a les respuestes posibles pa cuatro grandes entrugues alredor de los PEVA: ¿Cuálos son los sos elementos fundacionales y constitutivos? ¿Cómo se desendolquen y caltienen? ¿En qué midida consiguieron los efectos esperaos? ¿Qué porvenir tienen?

Nel desenvolvimientu d'estes entrugues d'investigación, puen estremase aspectos cualitativos (caracterizar, describir, singularizar, clasificar, esaminar, esplorar), cuantitativos (midir, cifrar, cuantificar, evaluar numbéricamente) y otros más xenéricos (como recoyer o definir). En tou casu, descártase pa esti trabayu cualquier propuesta basada na confirmación o refuga d'hipótesis, que sedría dafechu apriorística pola falta d'antecedentes d'investigación. Abulta muncho más amañosu un diseñu non esperimental (ensin manexu de variables nin intervención sobre la realidá) y mestu (Fàbregues et al. 2016: 112), de calter descriptivu y non causal (Riba et al. 2011). El dispositivu metodolóxicu propuestu consiste na integración de dos ferramientes configuraes pa la producción de datos empíricos y estadísticos, cualitativos y cuantitativos. Per un llau, un averamientu etnográficu pente medies de la observación y la entrevista. Pel otru, un estudiu evaluativu al traviés d'un cuestionariu. El periodu estudiáu (1996-2019) correspuende al intervalu que va dende'l que pue considerase'l puntu d'inflexón na medra de la dixitalización n'Asturies y nel mundu hasta'l momentu d'execución del mesmu trabayu de campu.

Arriendes de trabayar namás con persones adultes non espuestes a riesgu especial, qu'ufrieron la so collaboración activamente y a les que s’informó de primeres a últimes, la investigación asumió los demás principios éticos de la European (2011).

\subsection{Ferramientes etnográfiques}

La producción de datos de calter empíricu pasa nesta investigación pel usu de dos ferramientes etnográfiques, la observación directa y la entrevista.

\section{Observación}

Gracies a una primer güeyada xeneral a les posibilidaes de formación en llinia de la llingua asturiana, estremárense, amás de dalguna propuesta 
parcial pasada o presente, los cuatro PEVA surdíos dientro del periodu estudiáu y que siguen, toos cuatro, n'activo:

- L’alministráu pola empresa Araz Net, identificáu na investigación col códigu AST.

- Col códigu INI, el que ye obra de l'asociación Iniciativa pol Asturianu.

- El dependiente de l'Asociación Faceira al que se-y conseñó'l códigu FAC.

- POS, xestionáu pol Institutu Asturianu d’Alministración Pública «Adolfo Posada».

El diseñu d'observación organízase dende la previsión d'un escenariu au l'investigador actúa como un alumnu, percorriendo dende dientro toles posibilidaes qu'ufre caún de los PEVA: lleendo, faciendo exercicios, interactuando nos espacios -si los hai- destinaos a ello, etc. Les categoríes d'observación dispunxéronse en tres grandes grupos d'alcuerdu cola tabla 1.

\begin{tabular}{|c|c|}
\hline \multicolumn{2}{|r|}{ Tabla 1. Categoríes d'observación } \\
\hline \multirow{2}{*}{$\begin{array}{l}\text { L'empiezu } \\
\text { y la fin }\end{array}$} & Entrada, rexistru, modalidaes, niveles. \\
\hline & $\begin{array}{l}\text { Evaluación final, certificación propia o qu'empobina pa una certificación ayena } \\
\text { pero correspondiente al nivel trabayáu. }\end{array}$ \\
\hline \multirow{3}{*}{$\begin{array}{l}\text { El proyectu } \\
\text { formativu }\end{array}$} & $\begin{array}{l}\text { Los campos principales de conteníu: espresión escrita, espresión oral, } \\
\text { comprensión llectora, comprensión auditiva, sociollingüística, usu artísticu de la } \\
\text { llingua. Dosificación, combinación. }\end{array}$ \\
\hline & Les fontes d'información: testos, imáxenes, vídeos, audiciones, xuegos, etc. \\
\hline & $\begin{array}{l}\text { Les actividaes: bayura, adaptación al nivel, información y conteníos. Presencia } \\
\text { d'autoevaluaciones y/o correcciones automátiques. }\end{array}$ \\
\hline \multirow[t]{2}{*}{$\begin{array}{l}\text { El fluyir } \\
\text { internu }\end{array}$} & $\begin{array}{l}\text { La navegación y la presentación: llinguaxe, axilidá, tresparencia, intuitividá, } \\
\text { visibilidá del conxuntu dende cualquier puntu, retornabilidá, índiz, aúda, } \\
\text { información de requisitos técnicos, posibilidá d'adaptar la navegación a los } \\
\text { gustos del alumnáu, diseñu, calidá de la llingua, actualización tecnolóxica. }\end{array}$ \\
\hline & Les aúdes esternes y la conexón del cursu con otres fontes. \\
\hline
\end{tabular}

\section{Entrevista}

La segunda de les ferramientes cualitatives d'esta investigación, con una configuración inspirada na observación previamente planificada y executada, ye una entrevista que, según Fàbregues et al. (2016), podía clasificase como llonxitudinal, retrospectiva, semiestructurada, individual, focalizada, en profundidá y directiva.

Les persones entrevistaes escoyéronse en ficies de recoyer les más de les miraes y vivencies. Pa ello, garantizóse que los 3 tipos de funciones desenvueltes 
tuvieren presentes nel estudiu de cada PEVA: la función promotora (P), centrada na concepción del programa y la so planificación global; la desenvolvedora (D), al traviés de la que se configuren o tresformen los PEVA nos planos pedagóxicu, filolóxicu o informáticu; y la docente (E), na qu'entren toles ocupaciones propies de les persones enseñantes: tutoría, actividaes, evaluación, ente más otres. A cuenta d'identificar toles entrevistes, conseñáronse col mesmu códigu que'l PEVA nel que tán y un orde de realización.

Pola concentración de funciones que desenvolvieron dalgunes de les 12 persones entrevistaes ( 3 en cada PEVA), la muestra representa un total de 22 funciones. Asina: l'informante Ast1 desenvolvió les funciones P y D, Ast2 (P, D y E), Ast3 (E), Ini1 (P, D y E), Ini2 (E), Ini3 (E), Pos1 (P), Pos2 (D y E), Pos3 (D y E), Fac1 (P y D), Fac2 (P, D y E) y Fac3 (D). Sabiendo que l'universu estadísticu yera de 16 persones (con 27 funciones desenvueltes), pue dicise que la muestra escoyida foi arrogante.

La entrevista compónse de 8 entrugues, caúna d'elles encontada en 5 cuestiones optatives pensaes p'audar a esplorar y p'algamar la mayor cantidá d'información, multiplicando les unidaes de recoyida hasta 40. Sicasí, la naturaleza d'esta ferramienta, obliga a prever el surdimientu d'otros datos non categorizaos o de rellatos qu'amiesten delles de les cuestiones plantegaes nel diseñu. Nesi sen, al envolcalu pal so estudiu inmediatu, cuadróse'l conteníu de les unidaes de recoyida coles categoríes analítiques diseñaes, conforme a lo dispuesto na tabla 2, au tamién se conseña pa qué funciones concretes van empobinaes les entrugues.

\begin{tabular}{|c|c|}
\hline \multicolumn{2}{|r|}{$\begin{array}{l}\text { Tabla 2. Entrugues y unidaes de recoyida de la entrevista, } \\
\text { formulaes como categoríes analítiques }\end{array}$} \\
\hline $\begin{array}{l}\text { Antecedentes, } \\
\text { contestu y surdimientu } \\
\text { del PEVA } \\
\text { (función P) }\end{array}$ & $\begin{array}{l}\text { Tipu d'entidá promotora } \\
\text { Persones implicaes y rellaciones dientro de la entidá } \\
\text { Oxetivos y funciones de la entidá } \\
\text { Principales actividaes de la entidá } \\
\text { Tresformaciones na entidá al desenvolver el PEVA }\end{array}$ \\
\hline $\begin{array}{c}\text { Razones } \\
\text { pa desenvolver } \\
\text { el PEVA } \\
\text { (funciones P, D y E) }\end{array}$ & $\begin{array}{l}\text { Razones tocantes a la normalización llingüística } \\
\text { Razones de tipu educativu } \\
\text { Razones culturales } \\
\text { Razones llaborales } \\
\text { Razones identitaries y otres }\end{array}$ \\
\hline $\begin{array}{c}\text { Planificación } \\
\text { del trabayu } \\
\text { (funciones P y D) }\end{array}$ & $\begin{array}{l}\text { Influyencies dientro y fuera d'Asturies } \\
\text { Oxetivos y fases } \\
\text { Públicu pal qu'empobina'l PEVA } \\
\text { Formatos d'aplicación previstos } \\
\text { Perspectives primeres }\end{array}$ \\
\hline
\end{tabular}




\begin{tabular}{|c|c|}
\hline $\begin{array}{c}\text { Medios } \\
\text { (funciones } \mathrm{P} \text { y D) }\end{array}$ & $\begin{array}{l}\text { Equipu humanu y perfiles } \\
\text { Tecnoloxía } \\
\text { Dotación económica } \\
\text { Collaboraciones esternes cola entidá promotora } \\
\text { Nuna situación ideal, medios deseables }\end{array}$ \\
\hline $\begin{array}{c}\text { Competencies de los } \\
\text { desenvolvedores } \\
\text { (funciones P, D y E) }\end{array}$ & $\begin{array}{l}\text { Competencia llingüística } \\
\text { Competencia educativa } \\
\text { Competencia dixital } \\
\text { Integración de competencies y coordinación } \\
\text { Claves d'ésitu, puntos fuertes, orixinalidá }\end{array}$ \\
\hline $\begin{array}{l}\text { Trabayu docente } \\
\text { (función E) }\end{array}$ & $\begin{array}{l}\text { Funciones de les persones enseñantes } \\
\text { Coordinación ente enseñantes } \\
\text { Aportaciones docentes al axuste/diseñu del PEVA } \\
\text { Esta vivencia docente en comparanza con otres estremaes } \\
\text { Dificultaes y problemes }\end{array}$ \\
\hline $\begin{array}{l}\text { Tresformaciones } \\
\text { (funciones D y E) }\end{array}$ & $\begin{array}{l}\text { Descripción y razones } \\
\text { Planificación de les tresformaciones } \\
\text { Influyencies } \\
\text { Rellación cola evolución personal-profesional del equipu } \\
\text { Previsión de tresformaciones futures }\end{array}$ \\
\hline $\begin{array}{l}\text { Evaluación global, } \\
\text { conclusiones } \\
\text { (funciones P, D y E) }\end{array}$ & $\begin{array}{l}\text { Evaluación cuantitativa } \\
\text { Evaluación comparativa con otres esperiencies asemeyaes } \\
\text { Evaluación comparativa cola evolución del enseñu virtual } \\
\text { Evaluación comparativa con cursos de llingües mayoritaries } \\
\text { Porvenir }\end{array}$ \\
\hline
\end{tabular}

\subsection{Ferramienta evaluativa}

$\mathrm{Pa}$ la parte evaluativa del dispositivu metodolóxicu, diséñase una ferramienta selectiva y estadística, tresversal y de busca (Riba et al. 2011), un cuestionariu que tien nel control de la fiabilidá de la estrapolación y na so consistencia interna (Fàbregues et al. 2016: 36) los principales argumentos de validez esterna.

La propuesta consiste en recoyer opiniones espertes d'individuos directamente implicaos nel enseñu del idioma, apurriéndo-yos primero les víes d'entrada a caún de los PEVA y pidiéndo-yos que los evalúen darréu de visitalos y probalos. La muestra estratificóse d'esta miente: formadores en cursos programaos fuera de los niveles reglaos (F), profesoráu de Llingua Asturiana en Primaria (M), en Secundaria (P), personal docente-investigador de la Universidá d'Uviéu (U) y persones emplegaes nos servicios de normalización llingüística $(\mathrm{N})$. Pa calcular el tamañu del universu a estudiar, dao que son plantiyes variables, fíxose la media arrendondiada de los valores 
máximos y mínimos rexistraos nos tres años postreros, cosa qu'iguó un total de 306 individuos: $\mathrm{F}=17, \mathrm{M}=188, \mathrm{~N}=76, \mathrm{U}=10$ y $\mathrm{N}=15$. Magar qu'un bon númberu de profesionales taben en dellos de los estratos, determinóse una adscripción namás (la más frecuente, intensa o actual).

Nel manexu de la muestra escoyóse una estratexa de minimización del error por estrapolacióm esterna (Fàbregues et al. 2016: 46) de los resultaos ( au $\mathrm{p}=\mathrm{q}=0.5$, marxe d'error $=0.05$ y nivel de confianza $=0.95$ ). L'oxetivu nel diseñu d'investigación yera qu'esti valor nun xubiere del $15 \%$ pa poder escoyer con seguranza estadística tolos resultaos superiores al 65\%. Al empar, y anque enxamás tuvo previstu un posible analís por estratos (implicaría velos como variable independiente), aseguróse l'equilibriu de la estratificación fixando un error por estrapolación máximu del $30 \%$ en cualquier estratu y una diferencia enxamás mayor al 5\% ente ellos. Al cabu, consiguíos 63 cuestionarios válidos $(\mathrm{F}=10, \mathrm{M}=19, \mathrm{~N}=18, \mathrm{U}=7$ y $\mathrm{N}=9)$, l'error por estrapolación pal conxuntu ameyoró muncho'l diseñu, con un $11 \%$, y l'equilibru de la estratificación quedo bien afitáu con un $20,8 \% \pm 0,5 \%$ nesti datu. Otramiente, en cata de l'aleatoriedá del estudiu, pidióse la collaboración al 100\% d'individuos nos estratos más pequeños ( $\mathrm{F}, \mathrm{U}$ y N), mentes qu'en $\mathrm{M}$ y $\mathrm{N}$ fixéronse 30 y 30 pidimientos elixíos al azar ente les 58 señes de corréu electrónicu compilaes en M y les 43 en N. Enantes d'ello, fixérase un llamáu públicu en redes sociales xenerales y especializaes.

En previsión de trabayar con una muestra bultable, consideróse amañoso traducir numbéricante la información y, pa ello, usóse una escala tipu Likert de 5 valores (au 0 ye «dafechu en desalcuerdu» y 4 ye «dafechu d'alcuerdu») en 115 de les 124 cuestiones qu'igüen el cuestionariu. Aparte, 4 entrugues garraron datos identificativos y otres 5 compilaron crítiques y/o propuestes. La tabla 3 recueye la organización xeneral d'esta ferramienta.

\begin{tabular}{|c|l|}
\hline \multicolumn{2}{|c|}{ Tabla 3. Secciones del cuestionariu } \\
\hline Primera & Identificación de la persona encuestada \\
\hline Segunda & Razones pa escoyer un PEVA (cuestiones 1-10) \\
\hline Tercera & $\begin{array}{l}\text { Comparanza ente los alumnaos de cursos de llingües minoritaries y } \\
\text { mayoritaries (cuestiones 11-15) }\end{array}$ \\
\hline Cuarta & Evaluación d'INI (cuestiones 16-40) \\
\hline Quinta & Evaluación de FAC (cuestiones 41-65) \\
\hline Sesta & Evaluación de POS (cuestiones 66-90) \\
\hline Séptima & Evaluación d'AST (cuestiones 91-115) \\
\hline Octava & Considerances de conxuntu (cuestiones 116-120) \\
\hline
\end{tabular}


En ficies de destacar delles idees xenerales, iguáronse unos indicadores conglomeraos. A partir de les secciones 2 y 3 de la ferramienta, diseñóse una primer xeneración d'ellos pa englobar en tres tipos los motivos polos que, al ver de les persones encuestaes, la xente quier formase al traviés d'un PEVA: identitarios (MI), llaborales (ML) y culturales (MC). Coles cuestiones correspondientes al estudiu detalláu de los PEVA (secciones 4 a 7) tresnáronse dellos indicadores más, tocantes a la calidá de dellos aspectos: conteníu (CON), actividaes (ACT), navegación (NAV), diseñu (DIS), sofitu pedagóxicu (SOF) y valoración global (IVA).

Como midimientu de la consistencia y la fiabilidá del cuestionariu, l'Alfa de Cronbach ufrió un nivel escelente $(\alpha=0.98)$, qu'inda foi más altu $(\alpha=0.99)$ amestando a les 115 cuestiones n'escala los 9 indicadores numbéricos calculaos a partir d'elles y que tán acabantes de presentase.

\section{AVERAMIENTU ETNOGRÁFICU}

Esta primer parte de la discusión de los resultaos, centrada nos datos empíricos, básase nel material producío coles dos ferramientes de mena etnográfica de la investigación: la observación y la entrevista. De primeres, propónense unes notes cronolóxiques del ámbitu estudiáu, pa siguir con una caracterización de los PEVA y zarrar con dellos apuntes alredor de los motivos y les circunstancies polos que surdieron y siguen n'activo güei.

\subsection{Notes pa una cronoloxía del asturianu y el so enseñu na rede}

Nel añu 1995 yá se rexistraben interacciones virtuales con vocación didáctica alredor de la llingua asturiana, comunicaciones de tipu metallingüísticu ente persones que taben fuera y dientro d'Asturies (informa Ast2) encontaes nuna d'aquelles primeres formes d'alcuentru dixital: les llistes de corréu. Al añu viniente diba entainase pel camín abiertu. Asina surdía Soc.Culture.Asturies, la edición asturiana d'otru tipu de llista d'Usenet, esta de distribución, a la que yá-y diben mandando dalgo otres llingües y cultures. De forma asemeyada, IRC (Internet Relay Chat) abriere la canal \#AsturiEs, que tamién diba promover el discutiniu, munches vegaes de calter interformativu, sobre l'asturianu (Ast1, Ast2). Per eses mesmes dates, Ast1 y Ast2, que víen cierta demanda pa ello, yá entamaren el trabayu pa crear AST, el primer PEVA, que diba apaecer n'avientu de 1997; meses primero, yá empecipiare l'actividá n'Asturia, el portal au añeró AST, pero tamién el buscador Úlos y la tienda Asturshop. Llingua-list, otra llista de corréu electrónicu más, esta sí específica pa falar del asturianu, complementó les 
funciones d'AST a lo llargo de los sos tres primeros años de vida, anque nun fore la so misión esclusiva y, de fechu, siguiere activa depués d'ello (Ast1, Ast2). En paralelo, otra ferramienta didáctica llegare a tresnase naquel añu 1997, pero nun cuayó igual qu'AST y acabó arrequexada nun espaciu nomáu La Fueya d'Asturies, que foi perdiendo visibilidá hasta desapaecer (Ast2).

Los años centrales de los noventa condensaron dellos fechos d'importancia sociollingüística y que son contestuales pal oxetu d'esti estudiu según informen Ast1, Ast2, Ast3, Ini3 y Pos2: la presencia del asturianismu na Xunta Xeneral (1991-1999), el primer casu de demanda d'oficialidá pal asturianu nun partíu estatal (Izquierda Xunida, elecciones de 1995), el Pactu pol Autogobiernu y la Oficialidá (1995), l'apaición del selmanariu Les Noticies (1996), les declaraciones d'oficialidá en dellos conceyos (dende 1997), l'aprobación de la Llei d'Usu y Promoción (1998) y l'asoleyamientu de la gramática normativa (GLLA) (1998). La reforma ensin oficialidá del estatutu d'autonomía d'Asturies (1999) frustra esti periodu d'esperances asturianistes, que remata tamién col arrampuñamientu de la llicencia a Radio Sele (1999) (Ini1), emisora dafechu n'asturianu que llevaba emitiendo dende 1986.

Dando continuidá a la tendencia esponencial na dixitalización de la sociedá nos noventa postreros, los cuatro primeros años del sieglu XXI siguen siendo tiempu de foros y buscadores, del espaciu web 1.0 y les sos páxines estátiques (resume Ast2). En rellación col enseñu y la presencia social del asturianu, namás va haber una novedá importante nesti periodu: l'apaición del Diccionariu de l'Academia de la Llingua Asturiana (DALLA) a finales de 2000 (Ast2, Pos3). Nel planu políticu, yá en 2003, el PSOE forma gobiernu con Izquierda Xunida - Bloque por Asturies y el papel institucional del asturianu crez (Ast3); al empar, surde'l primer serviciu de normalización llingüística d'un conceyu (Xixón) (Ast3, Ini1). Al otru añu, l'Institutu «Adolfo Posada» (con rangu de dirección xeneral del Gobiernu del Principáu d'Asturies) entama'l llabor pa formar el segundu PEVA n'orde cronolóxicu: POS, qu'entra en funcionamientu en 2005 dirixéndose de mano a les y los trabayadores del Principáu d'Asturies y, más alantre, a otros colectivos (Pos1, Pos2). Cuasimente al empar, apruébase'l que ye hasta güei l'únicu plan de normalización llingüística autonómicu (2005-2007), nel qu'hai cuatro acciones previstes nel dominiu formativu, una d'elles pensada pa POS (Pos1).

Coles emisiones de la Radiotelevisión del Principáu d'Asturies prácticamente escaecíes del asturianu dende'l so primer momentu (2006) n'opinión de dellos informantes, el segundu llustru del sieglu XXI trescurre ensin grandes novedaes pa la normalización y l'enseñu (Ini1, Fac1), quitando que col so inxerimientu nel espaciu européu, la Universidá d'Uviéu cambió los títulos propios d'espertu y especialista en llingua asturiana por una 
mención en maxisteriu y un mínor nes filoloxíes (Ast3, Ini1, Ini2). Nesi escenariu d'invariabilidá social del asturianu pero de máximu ritmu de dixitalización surden ferramientes como'l traductor y conxugador Eslema y les versiones virtuales del DALLA y del Diccionario General de la Lengua Asturiana.

Yá nesta segunda década del sieglu XXI, l'apaición d'INI y FAC, los PEVA caberos hasta güei, va ser la principal novedá nesti dominiu. Dende la nacencia d'Iniciativa pol Asturianu, los formadores de los cursos d'esta asociación taben mandándo-y abondo a la rede (Ini1, Ini2, Ini3) y namás unos meses depués (2012), fechu'l volcáu de conteníos a una plataforma d'aules virtuales, INI configurábase cuasique netu a como ye anguaño. Pel so llau, l'asociación lleonesa Faceira asoleya en 2012 el so manual de gramática Xurdir (Fac1, Fac2), qu'en 2013 diba complementase con actividaes, tresnando una combinación que, alministrada pela vía dixital, perfái lo que yera y ye FAC (Fac3).

Dende entós hasta agora -según apunten los protagonistes de los últimos PEVA desendolcaos-, el voluntarismu domina la xera d'atender toos estos programes igual que fai 22 años promovió l'entamu d'AST (Ast1, Ast2), cola mesma ruindá de medios y les mesmes dificultaes pa consiguir poner en manes especialistes estos desenvolvimientos (Fac2, Ini1, Ini2, Ini3). Nel llustru postreru, col emplegu intensificáu de tou tipu de redes sociales y espacios web 4.0, apaecen delles ferramientes qu'abren posibilidaes futures pal enseñu virtual del asturianu. Asina, envereden nesi sen: un cursu cenciellu na plataforma d'autodeprendimientu de llingües Memrise, una canal en Youtube con dalgunes pieces formatives o dellos perfiles de Twitter y Facebook nos que s'alderica o, inclusive, s'apurren conseyos d'usu del asturianu.

\subsection{Qué ye y qué nun ye un PEVA}

La idea de PEVA, como formatu pedagóxicu concretu que yá se presuponía nel diseñu d'investigación, ye bona de desllindar agora, a la lluz de los datos producíos etnográficamente. Al ver de los informantes, qu'usen soluciones como «cursu», «plataforma» o «programa», pa falar de lo qu'equí se conseñó como PEVA, han dase unes característiques básiques que valen de finxos nesti «desllinde». Un PEVA, entós, sedría siempre un productu con vocación de completitú nel oxetivu final d'alfabetizar o audar a ello, que tien teoría y práctica y una organización estructurada. Con estes cuestiones cuantitatives, una cualitativa: la formación ha tener una carga bultable, de delles decenes d'hores.

Dientro d'esta noción xeneral, la comprensión de la bayura de 
presentaciones de los PEVA, fai interesante clasificalos de delles maneres. Mirando la forma xurídica de les entidaes tres de los PEVA, atópense 2 asociaciones, 1 institución pública y 1 empresa. Les dos asociaciones, Iniciativa pol Asturianu (responsable d'INI) y Faceira (de FAC), nun recibieron aúda institucional nenguna, anque la primera d'elles cobra una tasa simbólica d'inscripción al PEVA. La empresa Araz Net (AST) sí vien algamando cuasi-añalmente dalguna aportación pública a la promoción del asturianu pol espaciu au s'inclúi'l PEVA (pero non específicamente pa elli). POS, dependiente del Institutu Asturianu d'Alministración Pública «Adolfo Posada» (que ye una entidá dedicada a la formación), finánciase directamente con un dineru público (Pos1) que nel so momentu d'entamu facilitó'l llabor profesional (al ver de Pos2 y Pos3) de, ente otro, una empresa tecnolóxica, dos actores qu'interpretaron delles situaciones didáctiques y dos maestros espertos n'asturianu qu'estructuraron y escribieron tolos conteníos.

Reparando na forma d'accesu y navegación, 2 PEVA son dafechu llibres y ensin sesión personalizada (FAC y AST) y 2 precisen de clave y rexistru d'usuariu (INI y POS). Estes mesmes dos pareyes caltiénense atendiendo pa la posibilidá de certificar la conocencia algamada al rematar y pal criteriu de cantidá de niveles ( 1 o 2), dalgo qu'informa de la fondura y la proyección de la propuesta pedagóxica d'INI, pero sobre manera de POS. Cola tutorización como criteriu, namás INI caltuvo siempre esta posibilidá, anque, en dellos momentos, tamién la tuvieron AST y POS, esti últimu d'una forma dafechu profesional. En cuantes a la collaboración con otres entidaes diferentes a les promotores pa dalguna fase del so desendolcu, dos PEVA tienen dalgún tipu d'esperiencia: INI (que caltién un alcuerdu de formación cola Academia de la Llingua Asturiana que ye estensivu al so PEVA) y POS (que, como se dixo, coordinó axentes mui estremaos na so creación).

El paisaxe interior observáu nestos PEVA y la so funcionalidá tamién son desiguales. D’una mano, la simplicidá d'AST y FAC favorez muncho la navegación, que ye intuitiva en dambos casos, anque la imaxe probe de FAC nun aúda a tener una esperiencia prestosa, cosa qu'AST sí ufre. N'INI, anque tien la ventaya d'usar una plataforma corriente y, poro, reconocible, rescamplen les anuncies publicitaries que torguen el fluyir pela páxina, mentes que'l diseñu, poco o nada personalizáu, resulta mui fríu, poco afayadizu. POS, que ye'l PEVA fundamentáu nel desendolcu de conteníos y formes más curiosu, tien anguaño un grave problema d'obsolescencia qu'amala la navegabilidá, condicionada por duplicao: pola plataforma base na que tán asitiaos los componentes y pola dependencia de la tecnoloxía Flash, que yá taba a piques de quedar desaniciada nel momentu de la observación equí fecha.

Pela boca de los informantes pue falase tamién d'una xenealoxía de 
los PEVA: Fac1, Fac2 y Fac3 reconocen la influyencia directa d'AST en FAC y toles otres persones entrevistaes afirmen conocer la posición pionera d'AST. Tan allargada cronolóxicamente ye la presencia d'esti PEVA, que nuna de les entrevistes (Pos3) rellatóse qu'esta mesma persona trabayare dacuando nel desendolcu, años primero, d'AST. Nesti aspectu temporal, hai que recoyer les evoluciones principales vivíes na forma de xestionar estos programes. En 1999, AST modificábase (Ast1) p'afayase nes novedaes normatives trayíes pola GLLA. N'ochobre de 2000 los exercicios del cursu pasen a ser d'autocorrección y hai un cambéu de tutor, que diba caltenese d'ehí a 2004 y, p'acabu, otru cambéu más de tutor d'entós a 2008, cuando'l cursu dexa de tenelu definitivamente (Ast2, Ast3). POS, depués d'unos años con diferentes tutores al cargu de la dirección pedagóxica del cursu, en 2011, metanes d'una gran crisis gubernamental y presupuestaria, dexa de tener esti sofitu (Pos3) pasando a ser, d'ehí al añu 2018 (cuando lu recupera), un cursu d'autodeprendimientu (Pos1). Otra de les grandes tresformaciones observaes nesti dominiu cinca tamién al desenvolvimientu de POS, que surdió como sitiu de formación n'asturianu pa les y los trabayadores de les instituciones públiques autonómiques (Pos2, Pos3) y acabó asumiendo otres aplicaciones: nes comunidaes asturianes na diáspora, na radiotelevisión, en dalgunos conceyos y tamién, inclusive n'abierto (Pos1), como MOOC ensin certificación.

\subsection{La dixitalización d'una voluntá colectiva}

En cuantes a la mena de públicu al que, según el xuiciu de los 12 informantes entrevistaos, queríen unviar los sos PEVA, l'alcuerdu ye total: les persones que se sienten movíes a deprender la llingua asturiana amosaríen posiciones afectives en diferentes graos, seya por «militancia» (Fac2) o porque-yos prestaría reapropiase d'un idioma que yá «ye de so» (Ast3) pero que nun dominen pola mor de les poques oportunidaes alfabetizadores que seyos presentaren (Ini2, Ini3, Fac1). Apuntóse tamién como motivu, anque con menos frecuencia, la necesidá de conexón cola realidá asturiana de les persones inmigraes (Ast3) y cuasique tolos informantes vieron como otra causa posible'l deséu de caltener la rellación «cola so cultura» en persones emigraes d'Asturies y Lleón. Cuasi la metá de los entrevistaos reconocieron que nel tiempu más recién hai ciertu enclín al espoxigue de la demanda basada nes esperances llaborales, sobre manera darréu de l'amplificación del discutiniu social alredor de la oficialidá del idioma. Otres razones más lligaes a la carrera profesional o a «facer curriculum vitae» atoparon apoyu, sobre too nes entrevistes tocantes a POS, un programa que, como yá se dixo, ta mui rellacionáu cola función pública dende la so concepción mesma. 
En xeneral, la competencia dixital de los promotores de los PEVA suel ser la más especializada y la menos tresversal y, asina, si en POS foi una empresa esterna la que desenvolvió esta parte, n'AST y en FAC namás contaron con una persona que pudiere encargase de la programación informática (Ast1 y Fac3). Pel so llau, INI, qu'asentó nuna plataforma preprogramada, nin siquier tuvo nengún especialista. Pela cueta, les competencies llingüística y docente apecen más compartíes dientro de los PEVA. Otramiente, fai falta conseñar que nes 12 entrevistes feches aseguróse que nenguna de les persones participantes tenía esperiencia nin formación específica n'e-learning nel momentu d'entamar el so PEVA, dalgo que, al ver de los informantes, tampoco nun camudó muncho na actualidá.

Tolos entrevistaos y, poro, protagonistes d'estos PEVA, tán contentos cola aportación realizada anque, n'INI, AST y FAC, apréciase una satisfacción relativa, basada nel reconocimientu de la humidá de medios y na correspondiente moderación del enfotu puestu nello. Anque satisfechos más pol aspectu cualitativu qu'implica la so propia presencia na rede, tamién rescamplaron cuantitativamente'l so impactu en tres de los PEVA, nos que dispunxeren dalgún mecanismu pa midir el númberu de persones que pasaron per ellos: AST, INI y POS (Ast1, Ini2, Ini3, Pos1, Pos2, Pos3).

Anque hasta nos trabayadores de la iniciativa más profesionalizada (POS) queden reconocíes dosis d'ilusión y voluntarismu estraordinaries a cuenta de la so rellación «militante» cola llingua asturiana (Pos2 y Pos3), nel restu esti calter inda s'intensifica (Ast1, Ast2, Ast3, Ini1, Ini2, Ini3, Fac1, Fac2 y Fac3). La so traducción inmediata ye la falta de tiempu disponible que dedica-y al PEVA (por entrar en disputa col so tiempu llaboral) y de tecnoloxía y d'estructures organizatives que son propies de los contestos profesionales y non asina de los informales. Como diz Ast2: «si llegamos a dedicanos a esto de verdá, agora tábemos falando d'otres coses». Xuntos nestes mesmes circunstancies, caltiénse l'alcuerdu de los informantes d'estos tres PEVA al sorrayar los dos grandes motivos qu'emburriaron a cada entidá promotora: el voluntarismu asociáu a la toma de conciencia del riesgu de desaniciu del idioma («a la militancia», diz de nueves Ast1) y l'interés por facer pública la esistencia de la llingua nuna sociedá dixitalizada, cosa que suel espresase en términos de «normalización llingüística» (Ast3, Ini2, Ini3, Fac1 y Fac2) menos nel casu de Fac1, que quier más velo como dalgo anterior a eso, como un glayíu d'esistencia, porque «ensin apoyu institucional nun pue falase de normalizar». Nesi sen, hai que rescamplar que les entidaes que promueven INI y FAC son asociaciones que se reconocen «en defensa» de los drechos de les persones que quieren falar esta llingua (Ini1, Fac2). 


\section{ESTUDIU EVALUATIVU}

Como yá s'espunxo nel diseñu metodolóxicu, la escoyeta de la muestra pal cuestionariu respuende a una idea d'estratificación, pero en nengún casu quixo facese del oficiu una variable independiente: por eso la esposición de datos que vien darréu namás apunta pal conxuntu de la muestra. En ficies de conocer les tendencies principales, calculáronse los porcentaxes algamaos por cada respuesta en caúna de les 115 cuestiones n'escala (de 0 a 4), pero tamién por estos dos conglomeraos xenerales: los desalcuerdos (que xunten los valores 0 y 1) y los alcuerdos (3 y 4), dexando nun marxe d'indefinición el valor 2, que yera'l central ente les opciones de respuesta.

Al querer analizar namás los resultaos con garantía d'estrapolación, esbilláronse pa esti estudiu evaluativu les cuestiones qu'algamaron un 62\% d'alcuerdu o de desalcuerdu. Esta decisión asegura un apoyu mayoritariu (d'ente'l $51 \%$, nel casu peor, y el 72\%, nel meyor) na cuestión seleccionada.

La primer parte del cuestionariu, que recueye los datos personales de los participantes, vuelve una información acordies coles idees d'estratificación equilibrada y d'aleatoriedá defendíes na escoyeta de la muestra. Asina, anque la edá media del conxuntu ye de 42.21 años $(S=10.03, C V=0.24)$ y la distribución por sexos ye de 34 muyeres y 29 homes, nos estratos estos datos axústense a la especificidá que representen: nos ámbitos más feminizaos (profesoráu de primaria y secundaria) configuráronse estratos con mayoría clara de muyeres, mentes que nos más masculinizaos (profesoráu universitario y de cursos non reglaos) asocede lo contrario. Coles mesmes, el profesoráu universitario ye'l más mayor (54 años) y el de primaria, como ye manifiesto, el más mozo (36).

Na segunda sección del cuestionariu, sobre los motivos de les persones que faen cursos d'asturianu según el ver d'estos colectivos, lleguen al 90\% d'alcuerdu «pa enantar la so cultura asturiana»y «por razones afectives o identitaries» (esta, con un $63 \%$ dafechu d'acuerdu). «Pa falalu o escribilu meyor» llega al $89 \%$ d'alcuerdu y «pa caltenelu» recueye un $78 \%$. «Por desixencia llaboral» tien, pela cueta, un $84 \%$ de desalcuerdu. Na tercer parte, au sigue estudiándose la demanda de los PEVA comparando l'alumnáu de cursos de llingües mayoritaries y minoritaries, toles cuestiones rexistraron alcuerdos consistentes. El 100\% cuida que nos cursos de llingües mayoritaries «hai un interés llaboral» en «busca de certificación», tando'l 92\% y el 90\% dafechu d'alcuerdu nun y n'otru entecomináu. Que nos cursos de llingües minoritaries «hai esmoliciones afectives, identitaries o patrimoniales» algama'l 89\% d'alcuerdu (70\% dafechu) mentes que «fáense p'acabar cultivándoles nel ámbitu personal y artísticu» aunió un 76\% d'alcuerdu y «suel haber un alumnáu yá rellacionao cola llingua a deprender, que quier alfabetizase», un $71 \%$. Coles respuestes d'estes dos secciones, calculóse que 
los motivos identitarios (MI) algamen el $84 \%$ d'alcuerdu y el $62 \%$ los culturales (MC), mentes que los llaborales (ML) saquen el 78\%, pero de desalcuerdu.

\begin{tabular}{|c|c|c|c|c|}
\hline ENUNCIÁU DE LA CUESTIÓN & INI & FAC & POS & AST \\
\hline El cursu trabaya abondo y bien la espresión escrita & 16 & 41 & 66 & 91 \\
\hline El cursu trabaya abondo y bien la espresión oral & 17 & 42 & 67 & 92 \\
\hline El cursu trabaya abondo y bien la comprensión llectora & 18 & 43 & 68 & 93 \\
\hline El cursu trabaya abondo y bien la comprension auditiva & 19 & 44 & 69 & 94 \\
\hline Los conteníos tán bien combinaos y dosificaos & 20 & 45 & 70 & 95 \\
\hline La información preséntase con llinguaxe amañoso & 21 & 46 & 71 & 96 \\
\hline El cursu tien información complementario de calidá & 22 & 47 & 72 & 97 \\
\hline Les actividaes son les afayadices y tán bien planiaes & 23 & 48 & 73 & 98 \\
\hline Hai bones actividaes collaboratives pa trabayar en rede & 24 & 49 & 74 & 99 \\
\hline Esiste un índiz o directoriu qu'informa y orienta bien & 25 & 50 & 75 & 100 \\
\hline La navegación ye áxil y vase bien d'unes partes pa otres & 26 & 51 & 76 & 101 \\
\hline Úfrese información sobre les necesidaes de software & 27 & 52 & 77 & 102 \\
\hline Tien una aúda manuable y bona d'atopar & 28 & 53 & 78 & 103 \\
\hline Llegar al cursu, empezalu y/o rexistrase ye cenciello & 29 & 54 & 79 & 104 \\
\hline Les coses tán asitiaes y funcionen de forma lóxica & 30 & 55 & 80 & 105 \\
\hline Pue axustase l'apariencia a les preferencies del alumnáu & 31 & 56 & 81 & 106 \\
\hline El diseñu ye afayadizu y favorez navegar y concentrase & 32 & 57 & 82 & 107 \\
\hline Esiste la posibilidá de trabayar de forma tutorizada & 33 & 58 & 83 & 108 \\
\hline Hai autoevaluaciones bien planiaes pa potenciar l'enseñu & 34 & 59 & 84 & 109 \\
\hline Úfrese una evaluación esterna que fai bon midimientu & 35 & 60 & 85 & 110 \\
\hline El cursu amuesa un asturianu bayurosu y correctu & 36 & 61 & 86 & 111 \\
\hline El cursu algama los meyores estándares tecnolóxicos & 37 & 62 & 87 & 112 \\
\hline El cursu algama los meyores estándares pedagóxicos & 38 & 63 & 88 & 113 \\
\hline
\end{tabular}

Na cuarta sección, dedicada al estudiu d'INI, tres cuestiones consiguieron numberos enforma sólidos na estadística global. La 36 (sígase pela tabla 4) algama'l 75\% d'alcuerdu, mentes que la 16 y la 23 saquen el 63\%. Sobre un 
máximu de 4 puntos, los indicadores globales pa INI son de 2.27 en conteníos (CON), 2.53 n'actividaes (ACT), 2.27 en navegación (NAV), 2.12 en diseñu (DIS) y 2.43 en sofitu pedagóxicu (SOF). El so índiz de valoración xeneral (IVA), midíu sobre 10, ye de 5.96 puntos $(S=1.76, \mathrm{CV}=0.30)$.

Nel apartáu viniente, pal analís de FAC, rexistráronse niveles altos de desalcuerdu: $81 \%$ na cuestión $44 ; 79 \%$ en 47 y $60 ; 76 \%$ en 58,49 y $42 ; 73 \%$ na cuestión $62 ; 65 \%$ en $56 ; 63 \%$ en $63 ;$ y $62 \%$ en 52 . Pela cueta, esiste un alcuerdu positivu y sólidu na cuestión 54, con un 79\%. Los parámetros xenerales de FAC foron: $\mathrm{CON}=1.43, \mathrm{ACT}=1.35, \mathrm{NAV}=1.99, \mathrm{DIS}=1.60 \mathrm{y}$ $\mathrm{SOF}=0.68$. Pel so llau, IVA algamó los 3.58 puntos $(S=1.53, \mathrm{CV}=0.43)$.

Yá na sesta sección del cuestionariu, sobre POS, toles cuestiones que presenten fuercia estadística son positives. Con un $87 \%$ d'alcuerdu alcuéntrase la númberu 66 ; un $84 \%$ tienen la 71 y la 86 ; un $83 \%$ la 84 ; un $79 \%$ la 68 ; un $78 \%$ la 73 ; un $76 \%$ la 70 y la 75 ; un $75 \%$ la 88 ; un $67 \%$ la cuestión 82; un $65 \%$ la 87 ; y un $63 \%$ la 80 y la 85 . Les cifres globales de POS son: $\mathrm{CON}=2.88, \mathrm{ACT}=2.73, \mathrm{NAV}=2.48, \mathrm{DIS}=2.70$ y $\mathrm{SOF}=2.66$. La valoración xeneral (IVA) foi un $7.58(S=1.60, \mathrm{CV}=0.21)$.

No tocante a AST, nel sépitmu apartáu, hai dellos alcuerdos globales en sen positivu. El 86\% (60\% dafechu d'alcuerdu) cola cuestión 104; el $81 \%$ cola 111 ; el $75 \%$ cola 101 ; el $71 \%$ cola 105 ; el $68 \%$ cola 91 ; el $65 \%$ cola 96; y el $62 \%$ cola 100. Obsérvense equí tamién informaciones estadístiques fuertes en sen negativu y, asina, el $78 \%$ ta en desalcuerdu cola cuestión 110; el 76\% cola 108; el 71\% cola 92; y el 62\% cola 99 . Les midíes conglomeraes p'AST son: $\mathrm{CON}=2.02, \mathrm{ACT}=2.20, \mathrm{NAV}=2.65$, $\mathrm{DIS}=2.42$ y $\mathrm{SOF}=0.78$. En xunto, IVA=5.55 $(S=1.37, \mathrm{CV}=0.25)$.

$\mathrm{Na}$ sección octava del cuestionariu, d'entrugues xenerales sobre los PEVA, hai desalcuerdos sonces (pal nuesu compromisu de bona estrapolación) nes cuestiones tocantes a la valoración global del desenvolvimientu tecnolóxicu y pedagóxicu (116 y 117) d'estos programes. Mentanto, la cuestión que cinca a la profesionalidá y a la modernidá de la imaxe de los PEVA (118) tamién collecha un resultáu dondu, pero positivu. L'impactu que los PEVA tienen sobre la normalización (119), sí entra bien nes cifres consideraes estadísticamente consistentes nesti estudiu, con un $79 \%$ d'alcuerdu (59\% dafechu d'alcuerdu).

Miraos en xunto, 14 de los 20 indicadores estudiaos (5 indicadores en caún de los 4 PEVA) tán «aprobaos» al consiguir 2 o más puntos. Na media individual d'estos 5 indicadores vese que, menos en $\mathrm{SOF}=1.64$, siempre se superen los 2 puntos, siendo'l máximu NAV=2.34. Más en xeneral tovía, la media d'IVA nos cuatro PEVA ye 5.67, quedando dos d'ellos cuasi nesi mesmu nivel de valoración 
(INI +0.28 y AST -0.12 ) y otros dos mui lloñe d'elli, unu perriba y otru perbaxo: POS (+1.91) y FAC (-2.09).

Lleendo comparativamente los 5 indicadores globales (ACT, CON, DIS, NAV y SOF), representaos pa los 4 PEVA na gráfica 1 , rescampla que POS algama les meyores puntuaciones en toos menos en NAV, que ye más altu n'AST; seguramente penalizáu POS polos problemes d'obsolescencia yá referíos y emponderáu AST pola so cenciellez. La posición más baxa pa los cinco indicadores ye la de FAC, mentes qu'INI ye'l segundu PEVA en tres indicadores y terceru nos otros dos. Ente los que quiciabes precisen de dalguna desplicación encontada nel averamientu etnográficu previu, el fechu de que POS algame la meyor puntuación en SOF namás unos meses depués de recuperar la tutorización (según Pos1, darréu de tar siete años ensin ella) seique informa de la bona calidá del serviciu. Del mesmu xeitu, qu'AST supere a INI en DIS, pue tar falando de que la so páxina simple pero llimpia ye más afayadiza que la d'INI, más complexa pero con publicidá. Na interpretación global de la gráfica 1, hai que se decatar de qu'un pentágonu grande indica un PEVA con IVA altu, mentes que la so regularidá señala bien l'equilibriu ente los indicadores que lu describen.

Igual les crítiques y suxerencies collechaes individualmente pa cada PEVA (nes cuestiones 40, 65, 90 y 115) que les referíes al conxuntu (120), estráinse d'esti analís cuantitativu pol so calter suxetivu, aguardando la síntesis final y la formulación de propuestes.

\section{Gráfica 1. Representación} pentagonal de los PEVA.

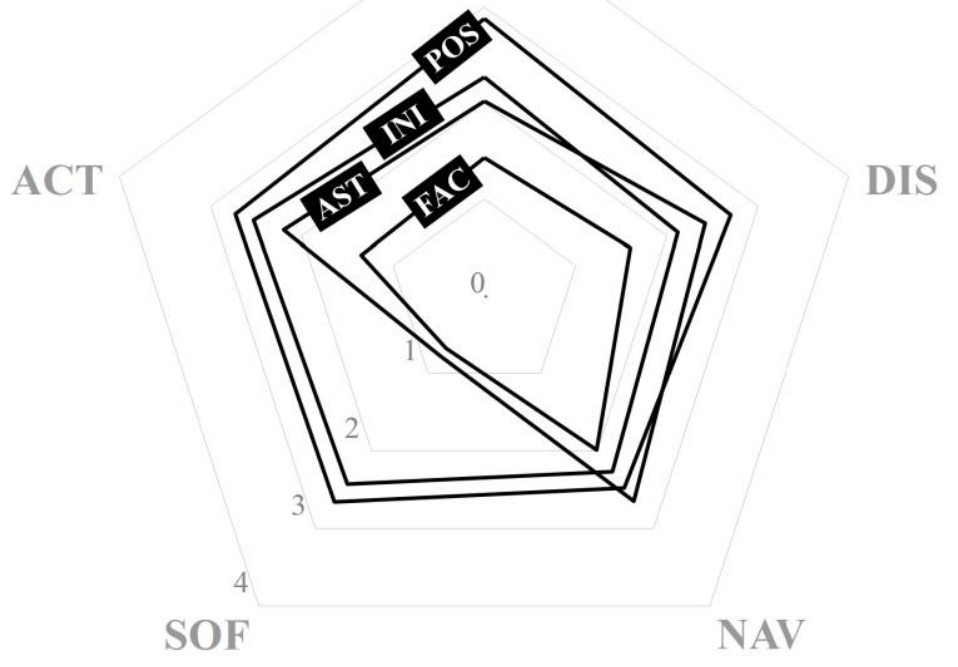




\section{SÍNTESIS}

Como se vio na revisión bibliográfica, la bayura llinguística del mundu esprésase na esistencia de más de 7.000 idiomes, de los que namás una parte pequeña son mayoritarios, alredor del $30 \%$ tán clasificaos en peligru de desaniciu y solo unos 100 tienen dalguna forma d'oficialidá (cálculos propios colos datos de Moseley 2010). Dende mediaos de los 90, cola xeneralización d'internet, munches d'estes llingües minoritaries emprimaron a usar la ferramienta dixital, desendolcando esperiencies sociales y educatives que, en dellos casos, llegaron a concretase en programes integrales pa deprendeles virtualmente. Estes acciones, igual qu'otres más xenerales o tresversales, inspiraron estudios compilatorios y conceptuales y propuestes metodolóxiques coles que s'afayar meyor nesta realidá educativa nueva.

$\mathrm{Na}$ intersección ente llingua asturiana y ferramienta dixital, la bibliografía apurre reflexones xenerales sobre les posibilidaes qu'internet-y ufierta a esti idioma, daqué estudiu tocante a los usos cotidianos na rede que fala n’asturianu y delles propuestes didáctiques dirixíes, más qu'al enseñu virtual como talu, al aprovechamientu de lo dixital na asignatura de Llingua Asturiana nos niveles reglaos. Poro, dende esi estudiu bibliográficu global y particular, pue concluyise que, al contrario que n'otros contestos, nel asturianu entá nun s'espublizaren obres allugaes nel dominiu concretu d'esta investigación. En señalando esta falta, consideróse acionao planiar un trabayu dirixíu a conocer fondamente l'enseñu virtual del asturianu, identificando los PEVA como estructures principales, anque ensin desdexar tol so contestu. La metodoloxía, ente lo etnográfico y lo evaluativo, sofítase en tres ferramientes: la observación, la entrevista y el cuestionariu. Tres de visitar el campu y d'analizar los datos empíricos y estadísticos tresnaos, apuerta'l momentu de sintetizalos pa, depués, facer dalgunes propuestes de futuru.

Los resultaos de la entrevista y del cuestionariu, amás d'ilustrar con mui bien de datos la situación d'amenorgamientu llingüísticu del asturianu que yá adelantare la bibliografía, enconten fuertemente qu'estes formaciones son atrayibles pa un públicu movío por causes de tipu identitariu, que quier deprender esti idioma declaráu en peligru manifiestu de desaniciu en ficies de normalizalu socialmente y de da-y vitalidá y continuidá. Y ye que, a los datos empíricos estrayíos de la entrevista alcuéntrase-yos bona correspondencia nes cifres altes del indicador MI del cuestionariu, igual que na gran consistencia algamada por toles cuestiones que nesta ferramienta metodolóxica indiquen la carga afectiva del posible alumnáu d'estos cursos y la so estremadura clara del que se forma en llingües mayoritaries.

Del otru llau, toles persones que promovieron, desendolcaron y punxeron 
escuela nos PEVA tán rellacionaes d'una manera o d'otra cola defensa, l'estudiu o l'espardimientu de la llingua asturiana. Nel casu de 3 de los 4 programes, la so apaición dióse cuasimente cola única enerxía del marcáu voluntarismu de los sos entamadores, enfotaos en normalizar socialmente l'asturianu per aciu de la so presencia en llinia y n'aumentar el númberu de persones alfabetizaes. En resultes, bien podía axetivase d'etnodidáctica esta coincidencia fuerte de discentes y docentes nes razones d'identidá llingüística colectiva como motor d'esti alcuentru formativu na rede.

Cola investigación confírmase tamién qu'igual los axentes implicaos nos PEVA que les persones dedicaes al estudiu, l'enseñu y la normalización del asturianu, consideren qu'estos programes tienen un efectu claru y positivu na proyección social del idioma. Otru elementu, si asina quier ser vistu, qu'empata colo identitario, lo colectivo y, de dalguna miente, «lo militante».

Los cuatro PEVA rexistraos y estudiaos entamáronlos y caltuviéronlos entidaes con formes xurídiques estremaes: dos asociaciones (INI y FAC), una empresa (AST) y una institución pública (POS). Dos cursos (FAC y AST) tán abiertos too siguío y ensin rexistru, nun empobinen pa nenguna certificación y tienen un nivel únicu. Pela cueta, nos otros dos (INI y POS) navégase baxo clave y con una sesión personalizada, empobinen pa unes certificaciones concretes y tienen dos niveles. Como se dixo, en xeneral, nel desendolcu de los PEVA los medios económicos foron ruinos y la planificación, precaria, nun siendo la bona y bayurosa concepción de POS, programa que, sicasí, nel momentu de la investigación yá rexistraba una baxada del so potencial formativu pola falta de continuidá na inversión nel plazu llargu, con un segundu nivel mui perbaxo del primeru, una cayida de la figura tutorial de 7 años y un software a piques de colapsar.

El primer PEVA, AST, apaeció en 1997 depués d'un tiempu d'esperiencies d'intercambéu llingüísticu y didácticu ente usuarios de la rede. Muncho más sero diben dir apaeciendo los demás, nesti orde: POS (2005), INI (2012) y FAC (2013). Hasta güei l'únicu PEVA que rexistró tresformaciones bultables na so redacción foi AST, que les tuvo por ampliación y por afayase meyor na normativa llinguística. Dende'l puntu de vista funcional, AST y POS vivieron la mesma variación: perder la tutorización (el primeru definitivamente y el segundu, una temporada). Anguaño, la busca etnográfica constata que nun se manifiesta escenariu de cambéu nengún pa los PEVA (naide nun prevé que camuden los formatos, nin qu'apaeza o desapaeza dalgún programa, nin nenguna otra acción tresformadora planificada nesti dominiu). Depués d'un cuartu de sieglu d'esperiencies dixitales pal enseñu de la llingua asturiana, los sos elementos constitutivo-fundacionales caltiénense, n'esencia, invariables. Asina, nun paez que los informantes aprecien 
variaciones sustantives no que fai a la situación sociollingüística del idioma nin no tocante a los medios materiales disponibles. Tampoco nun se manifiesten avances tecno-pedagóxicos importantes nes persones protagonistes de los PEVA, nengún casu de formación específica qu'empobine escontra desendolcos meyores nel futuru, nin tampoco grandes resultaos n'otres iniciatives parciales de tipu formativu que van apruciendo, ensin mayor inversión d'esfuerzu, a la busca d'impactu n'escenarios tan estremaos, y al empar tan asemeyaos nes sos formes d'interacción como son Youtube, Facebook, Twitter o Memrise, por dicir dellos.

Nel analís de los cuatro PEVA que lleven la parte mayor d'esti trabayu, viose que POS ye l'únicu qu'algama l'equivalente a un notable. Los datos estadísticos dexaron ver que l'alcuerdu positivu llega en POS a una gran parte de les cuestiones analizaes: los conteníos pa la competencia escrita y llectora, la so combinación y dosificación xeneral, lo afayadizo del llinguaxe emplegao, les actividaes no tocante a la so correspondencia colos oxetivos del cursu, el directoriu que facilita la navegación, l'allugamientu intuitivu y lóxicu de los elementos na pantalla, el diseñu, les autoevaluaciones, la posibilidá d'evaluase esternamente, l'usu d'un asturianu rico y unos bonos niveles tecnolóxicos y pedagóxicos xenerales.

Col so aprobáu, abondante nel primer casu y dalgo más ruinu nel segundu, INI y AST son los dos PEVA que queden en valoraciones cercanes a la media, anque con una distribución mui estremada. N'INI, dientro del cuestionariu, toles respuestes estadísticamente fuertes son positives, rescamplando'l bon emplegu del idioma, los conteníos pa la espresión escrita, la correspondencia ente oxetivos y actividaes y la información del cursu. Los sos indicadores, mui equilibraos, queden ente'l «aprobáu ruinu» de DIS y el «aprobáu altu» d'ACT. N'AST, pel so llau, alcuéntrense les polaridaes más marcaes del estudiu evaluativu, esistiendo siete alcuerdos y cuatro desalcuerdos enforma consistentes. Asina, AST sal bien en cuantes a los conteníos pa la escritura, la claridá del llinguaxe, la esistencia d'un directoriu, l'axilidá de la navegación, la cenciellez de la entrada y del empiezu del cursu, la colocación de los elementos y l'usu d'un bon asturianu. Pela cueta, recibe castigos, en forma de desalcuerdos, nos conteníos encaminaos a trabayar la espresión oral, nes actividaes collaboratives y na supervisión y la evaluación esternes. Reflexu d'esta inestabilidá nos resultaos nes cuestiones ye que, dientro de los indicadores globales, tea ente raspiar el notable en NAV y un suspensu duru en SOF (probando que malpenes queda niciu de cuando taba dotáu de tutorización).

En FAC, que ye l'únicu PEVA que suspende, y failo con claridá, el cuestionariu volvió un alcuerdu positivu («llegar y escomenzar el cursu ye 
cenciello») y diez negativos: conteníos pa la espresión oral, pa la comprensión auditiva, información complementaria, actividaes collaboratives, información sobre les necesidaes técniques, posibilidá d'axuste de la navegación, esistencia de supervisión, posibilidá d'evaluación esterna, nivel tecnolóxicu xeneral y nivel pedagóxicu xeneral. La so cenciellez ta a piques de da-y un «aprobáu» nel indicador NAV que ye'l más altu de los sos cinco conglomeraos. Pela cueta, SOF da-y un suspensu enforma duru.

Vistos en xunto, los datos empíricos y estadísticos informen de que los PEVA desendolcáronse, en xeneral, anque con escepciones perriba y perbaxo, nunos valores intermedios aceptables según el ver de les persones que fixeron el cuestionariu, pero tovía alloñaos de los escenarios óptimos y d'escelencia polos que naguaben dalgunos de los informantes y promotores entrevistaos. De xuru, esa moderación nos resultaos algamaos polos PEVA ye la que nun dexa ver, por casu, alcuerdos o desalcuerdos estadísticamente sólidos nes cuestiones qu'entruguen pola calidá de los desendolcos técnicu, pedagóxicu y estéticu. Sicasí, una d'estes cuestiones de conxuntu sí algama consistencia pa la media global, la que fala de la contribución positiva de los PEVA a la normalización social del asturianu, reflexón que, hai que s'alcordar, yá facíen toles persones entrevistaes.

\section{Propuestes de FUturu}

Respondiendo a ún de los oxetivos de la investigación, van formulase darréu dalgunes propuestes de futuru no tocante al campu estudiáu. Nesi sen, les que de siguío van presentase, ye menester introduciles al traviés de dos principios: primeru, que surden como enunciáu opuestu y tamién afirmativu de les que davezu sedríen les conclusiones y, segundu, qu'habríen mirase, si llega'l casu, enxamás aisladamente, sinón xunto al analís de resultaos recién espuestu, polo que pueda tener esti d'ilustrativu a la vista de los propios PEVA, como quien observa la representación esquemática d'un paisaxe delantre del mesmu paisaxe representáu, que-y lu resume y comenta.

Una de les grandes cuestiones que l'estudiu pon enriba la mesa ye la especificidá, pol so matiz identitariu no llingüístico, de la ufierta y la demanda del enseñu virtual del asturianu. Paez esti un asuntu trescendente pola dificultá que nel futuru pudiere suponer alcontrar l'equilibriu ente alliniase con esta característica - tan cercana al compromisu- de la que resalva, al empar, el voluntarismu que vien alloñando de tou profesionalismu a los más de los PEVA y a toles otres iniciatives asemeyaes y parciales equí investigaes. Esi enfotu militante que valió pa poner los finxos d'un dominiu nuevu fai cuasimente 25 años ye'l qu'agora, viendo los datos etnográficos, pue 
entendese como una torga si nun se complementa col inxerimientu acionáu de medios y planificación.

Nel ámbitu de los conteníos d'estos programes formativos, una demanda vieno repitiéndose na síntesis de resultaos d'esta investigación: ye necesario que cualquier meyora o novedá ente los PEVA tenga mui en consideranza la bona presencia de la espresión oral o ameyorar el so nivel, según el casu. En cuantes a la ferramienta cola que se trabayen conteníos y actividaes, hai delles víes de meyora que proponer. La más frecuente fala d'usar con determín lo audiovisual en toa clas de momentos. Apostar pol sofitu pedagóxicu per aciu de tutoríes cercanes y atentes, y tamién d'autoevaluaciones qu'animen al estudiu, ye otra de les llinies que propunxeron bien de cuestionarios y que ye obligao redactar equí. Ye preciso tamién caltener llimpies les pantalles d'elementos cafiantes que torguen la navegación y facer que la base gráfica, más que guapa, seya prestosa, y más que viviega, favoreza la concentración y allargue'l tiempu de conexón que l'alumnáu ye quien a dar. De forma asemeyada, propónse con repetitividá que les programaciones informátiques seyan cencielles, refugando les que s'empapicen o ralenticen el pasu pel cursu y tamién les que precisen de continues actualizaciones de software, escoyendo meyor les más llixeres y durables. Otra demanda repetida delles vegaes ye l'aumentu d'exercicios interactivos n'espacios virtuales, seyan ad hoc o configuraos con preseos corrientes na actualidá: corréu electrónicu, redes sociales xenériques, aplicaciones de tipu didácticu y la nube.

Nun capítulu, si se quier, estratéxicu, la investigación volvió coincidencies que bien valen pa redirixir la planificación futura del enseñu virtual del asturianu. D'una o d'otra miente, lo que relluma nesti puntu ye la necesidá de camudar l'actual marcu, caracterizáu pol allancamientu del llustru últimu, pa volver a unos niveles de plena funcionalidá lo desendolcao hasta agora, que nel so momentu valió pa poner l'asturianu ente les llocomotores pero qu'agora lu asitia pente los vagones caberos nesti metafóricu tren de les llingües minoritaries virtualizaes. Pa ello, a xuiciu de los informantes, son importantes tres coses básiques que se presenten encadenaes: superación del voluntarismu, multiplicación de medios económicos pela vía pública (con aúdes a empreses y asociaciones o executando los proyectos directamente nes instituciones) y coordinación d'especialistes y toa mena d'entidaes implicaes baxo una acción planificada, nel meyor casu, dende dalguna instancia del gobiernu asturianu. En ficies de xenerar les condiciones pa qu'aporte'l momentu, la investigación dexa una riestra d'idees a estudiar. D'un llau, la necesidá d'integrar y organizar estes formaciones virtuales dientro o en rellación a toles certificaciones esistentes, como ye'l casu del Marcu Européu, igualando tamién los niveles o equivalencies y les cargues llectives. D’otru, proyectar estos programes dende un criteriu d'autoridá y creitu non solo en 
mires del valir de la posible certificación, sinón tamién pola calidá y la confiabilidá llingüística que, al ver de los informantes, demanda davezu l'alumnáu: una llingua correcta, real y prestosa, que nun dea sitiu a la dulda nel procesu d'alfabetización de la persona neofalante, que dacuando ye la qu'atopa les faltes y les incoherencies de la propia propuesta pedagóxica.

Zarrando esti espaciu de propuestes, nun son menos importantes les munches idees apurríes pela vía etnográfica sobre la posible coordinación ente instituciones pal desendolcu de nueves esperiencies nesti ámbitu. La Universidá d'Uviéu, delles direcciones xenerales y conseyeríes del Gobiernu del Principáu y l'Academia de la Llingua Asturiana son les más apellaes na formulación hipotética d'estremaes plataformes conxuntes que nun dexen de ser la espresión múltiple del enfotu puestu en ver un tiempu nuevu prestixosu, profesional, académicu y funcional pal enseñu virtual del asturianu, que paez ser tanto como dicir, en xeneral, pal asturianu.

\section{Abreviatures}

Siguiendo l'orde d'apaición:

PEVA Programa d'enseñu virtual del asturianu.

AST PEVA allugáu n'Asturies.com y promovíu pola empresa Araz Net.

INI PEVA entamáu y esplotáu pola asociación Iniciativa pol Asturianu.

FAC PEVA entamáu y esplotáu pola asociación Faceira.

POS PEVA promovíu pol Institutu Asturianu d’Alministración Pública «Adolfo Posada».

P Función promotora dientro d'un PEVA (promotor/a).

D Función desenvolvedora dientro d'un PEVA (desenvolvedor/a).

E Función docente dientro d'un PEVA (enseñante).

F Estratu de la muestra estadística: docentes en formaciones non reglaes.

M Estratu de la muestra estadística: maestres y maestros de primaria.

P Estratu de la muestra estadística: profesoráu de secundaria.

U Estratu de la muestra estadística: personal docente-investigador universitario.

$\mathrm{N} \quad$ Estratu de la muestra estadística: trabayadores de servicios de normalización llingüística.

MI Índiz de motivos identitarios (máximu: 4).

ML Índiz de motivos llaborales (máximu: 4).

MC Índiz de motivos culturales (máximu: 4).

CON Índiz de valoración de los conteníos (máximu: 4).

ACT Índiz de valoración de les actividaes (máximu: 4).

NAV Índiz de valoración de la navegación (máximu: 4).

DIS Índiz de valoración del diseñu (máximu: 4).

SOF Índiz de valoración del sofitu docente (máximu: 4).

IVA Índiz de valoración xeneral (máximu: 10).

Fac2 Exemplu d'informante: entrevistáu númberu 2, correspondiente a FAC.

GLLA Gramática de la Llingua Asturiana.

MOOC Masive open on-line course.

$S \quad$ Desviación típica.

CV Coeficiente de variación. 


\section{BibLIOGRAFÍA}

ACADEMia de la Llingua Asturiana (2018): Informe sobre la llingua asturiana. Uviéu, Academia de la Llingua Asturiana.

ARnaU, J. (2005): «Nuevos retos en la enseñanza de la lengua minoritaria», en Llingua y lliteratura nes aules. Actes del X Alcuentru «Llingua minoritaria y educación». X.A. González-Riaño (coord.). Uviéu, Academia de la Llingua Asturiana: 213-229.

Asociación para la Investigación de Medios de Comunicación, AIMC (2018): Audiencia de Internet, abril-mayo 2018. Madrid, AIMC.

AssinI, A. (2014): «New resources for endangered languages», en MultiLingual 25-7: 28-30.

BERROS, X. (2007): «Lleendo y navegando col asturianu», en Recursos y estratexes didáutiques. X.A. González Riaño (coord.). Uviéu, Academia de la Llingua Asturiana, 35-42.

- (2008): «Llingua asturiana y competencia dixital: un retu pal profesorau», en Revista El Busgosu 7: 60-63.

Clark, L. (2008): «Cyberspace and Ethnic Identities: The Creation of Virtual Communities, the Case of Italy», en Cultural Education-Cultural Sustainability: Minority, Diaspora, Indigenous and Ethno-Religious Groups in Multicultural Societies. Z. Bekerman \& E. Kopelowitz (eds.). New York, Routledge: 383-398.

ClotheY, R.A. (2017): «The Internet as a tool for informal education: a case of Uyghur language websites», en Compare: A Journal of Comparative and International Education 47-3: 344-358.

CoRmack, M. (2005): «The Cultural Politics of Minority Language Media», en The International Journal of Media and Cultural Politics 1: 107-122.

CRU, J. (2015): «Language revitalisation from the ground up: promoting Yucatec Maya on Facebook», en Journal of Multilingual and Multicultural Development 36-3: 284-296.

CunLiFFE, D. (2007): «Minority Languages and the Internet: New Threats, New Opportunities», en Minority Language Media: Concepts, Critiques and Case Studies. M. Cormack \& N. Hourigan (eds.). Clevedon, Multilingual Matters: 133-150.

CunlifFe, D., M. HenRY, F. CARROLl \& R. KoP (2018): «Learning a minority language through authentic conversation using an online social learning method», en Computer Assisted Language Learning 31-4: 321-345.

CUNLIFFE, D. \& S. HERRING (2005): «Introduction to Minority Languages, Multimedia and the Web», en New Review of Hypermedia and Multimedia 11-2: 131-137.

De GraAf, T., C. VAn DER MeER \& L. Jongbloed-FABer (2015): «The use of new technologies in the preservation of an endangered language: The case of Frisian», n'Endangered languages and new technologies. M. C. Jones (ed.). Cambridge, University Press: 141-149.

Dooly, M. (2010): «Empowering Language Minorities through Technology: Which Way to Go?», n'ELearning Papers 19.

Dooly, M., C. Vallejo \& V. Unamuno (2009): Linguistic Minorities Thematic Report. Educational Policies that Address Social Inequality (EACEA Action 6.6.2). London, IPSE.

Edwards, V., L. Pemberton, J. Knight \& F. Monaghan (2002): «Fabula: A bilingual multimedia authoring environment for children exploring minority languages», en Language Learning \& Technology 6-2: 59-69.

EISENLOHR, P. (2004): «Language revitalization and new technologies: Cultures of electronic mediation and the refiguring of communities», n'Annual Review of Anthropology 33: 21-45.

EuRopean Science Foundation \& All EuRopean ACAdEMIES (2011): The European Code of Conduct for Research Integrity. Strasbourg, Ireg.

EvAS, J.C. (2014): «Minority languages fight for survIVA in the digital age», en The Conversation 17-02-2014.

FÀbregues, S., J. Meneses, D. Rodríguez-Gómez \& M. H. Paré (2016): Técnicas de investigación social y educativa. Barcelona, Editorial UOC. 
Ferré-Pavia, C., I. Zabaleta, A. GutiérreZ, I. FernándeZ-Astobiza \& N. Xamardo (2018): «Internet and Social Media in European Minority Languages: Analysis of the Digitalization Process», n'International Journal of Communication 12: 1065-1086

GALÁN, I. (2014): «L'asturianu y les nueves teunoloxíes: datos d'una encuesta d'usos llingüísticos», en Lletres Asturianes 110: 207-226.

GALLA, C. (2016): «Indigenous language revitalization, promotion, and education: function of digital technology», en Computer Assisted Language Learning 29-7: 1137-1151.

GARCÍA, X. \& R. SÁNChEZ (2008): Dos visiones asturianes de les nueves tecnoloxíes. Xixón, Araz Net.

GarCíA-ARIAS, X.L. (1976): Llingua asturiana y sociedá. Hestoria, entamos, enfotos. Uviéu, Conceyu Bable.

GonZÁleZ, M.T. (2014): «Los peligros del mundu dixital», en V Día de les Ciencies Asturianes. Uviéu, Gobiernu del Principáu d'Asturies: 37-66.

GonZÁlez-Riaño, X.A., X. García \& D. Melendi (2010): «Estudiu sobro l'emplegu del DALLA on-line. Un averamientu sociollingüísticu», en Lletres Asturianes 102: 71-85.

GRUFFYD, E.H. (2013): «Permeable and Impermeable Linguistic Boundaries: From Mass Media to Social Media in Policy and Practice in Minoritised», en Zer 18-35: 29-45.

GuARDADO, D. (2002): «La llingua asturiana na rede. Aplicaciones didáutiques», en Les habilidaes llingüístiques nel aula. Actes del VIII Alcuentru «Llingua Minoritaria y educación». X.A. González-Riaño (coord.). Uviéu, Academia de la Llingua Asturiana: 43-52.

HOGAN-BRUN, G. (2011): «Language planning and media in minority language and plurilingual contexts», en Current Issues in Language Planning 12-3: 325-329.

HugO, R. (2015): «Endangered languages, technology and learning: Immediate applications and long-term considerations», n'Endangered languages and new technologies. M.C. Jones (ed.). Cambridge, University Press: 95-110.

IgLesias, Y. \& M.E. Yeguas (2014): «El viaxe de les palabres pela rede», en V Día de les Ciencies Asturianes. Uviéu, Gobiernu del Principáu d'Asturies: 13-34

Johnson, I. (2013): «Audience Design and Communication Accommodation Theory: Use of Twitter by Welsh-English Biliterates», en Social Media and Minority Languages. Convergence and the Creative Industries. E. Jones \& E. Uribe-Jongbloed (eds.). Bristol, Multilingual Matters: 99-118.

JONES, A. (2015a): «Mobile Informal Language Learning: Exploring Welsh Learners' Practices», n'ELearning Papers 45.

- (2015b): «Social media for informal minority language learning: exploring Welsh learners' practices», en Journal of Interactive Media in Education 1-7: 1-9.

KeMP, S. (2018): Digital in 2018. Headline Internet, Social Media and Mobile Use Data for Every Country in the World. 〈http://wearesocial.com>.

MAC UidHILIN, N. (2013): «Learning communities mediated through technology: Pedagogic opportunities for minority languages", en Social Media and Minority Languages. Convergence and the Creative Industries. E. Jones \& E. Uribe-Jongbloed (eds.). Bristol, Multilingual Matters: 146-158.

McAllister, F., A. BlunT \& C. PRYs (2013): Exploring Welsh Speakers' Language Use in Their Daily Lives. Cardiff, Beaufort Research.

Morán, P. (1995): Llingua, información y nueves teunoloxíes. Mieres del Camín, Editora del Norte.

- (coord.) (2004): Informe y recomendaciones sobre la lengua asturiana. Liège, International Committee for the Safeguarding of Linguistic Rights in Asturies.

Moriarty, M. (2011): «New Roles for Endangered Languages», en The Cambridge Handbook of Endangered Languages. P. Austin \& J. Sallabank (eds.). Cambridge, University Press: 446-458.

Moseley, C. (ed.) (2010): Atlas de las lenguas del mundo en peligro (3a edición). París, 
Ediciones UNESCO.

NÚÑEZ, L.A. (2000): «Les nueves tecnoloxíes de la comunicación: la so importancia p'Asturies», en DD.AA, Dende Roma hasta Internet (seis visiones d'Asturies). Xixón, Fundación Nueva Asturies: 71-84.

ODÉ, C. (2008): «Teaching materials on language endangerment, an interactive e-learning module on the internet», n'Endangered Languages and Language Learning: proceedings of the conference FEL XII 4-27 September 2008 (2). Ljouwert/Leeuwarden, Fryske Akademy: 147-150.

OSTLER, N. (2001): «What is this technology ever going to do for minority languages?» n'ELSNEWS (Newsletter of European Network for Human Language Technologies 10-1: 6-7.

OvidE, E. (2005): «L'emplegu de les nueves teunoloxíes na enseñanza del asturianu", en Llingua y lliteratura nes aules. Actes del X Alcuentru «Llingua minoritaria y educación». X.A. González-Riaño (coord.). Uviéu, Academia de la Llingua Asturiana: 55-74.

PARICIO, S. \& J. MARTínEZ (2010): «Noves vies de revitalització per a llengües minoritzades: la repercussió d'internet en el cas de l'aragonès», en Digithum. Les humanitats en l'era digital 12.

RAU, V. \& Y. MENG-ChIEN (2007): «E-learning in Endangered Language Documentation and Revitalization», en Documenting and Revitalizing Austronesian Languages. V. Rau \& M. Florey (eds.). Honolulu, University of Hawaii Press: 111-133.

REERSHEMIUS, G. (2017): «Autochthonous heritage languages and social media: writing and bilingual practices in Low German on Facebook», en Journal of Multilingual and Multicultural Development 38-1: 35-49.

Riba, C.E., A. Fornieles, J. Turbany, A. Cosculluela \& J. Meneses (2011): Tècniques d'anàlisi de dades. Barcelona, UOC.

SAURí, R. (2004): «Un corpus pal asturianu. Les tecnoloxíes llingüístiques na consolidación de les llingües minorizaes», en Revista de Filoloxía Asturiana 3\&4: 135-174.

SEDILES, D. (2018): «Del aragonés al asturiano: cómo es aprender una lengua en peligro de extinción en pleno siglo XXI», en Magnet 11-11-2018.

SimONS, G. F. \& C.D. FENNIG (eds.) (2018): Ethnologue: Languages of the World, Twenty-first edition. Dallas, Texas, SIL.

SLimane, M. (2008): Appropriating new technology for minority language revitalization: The Welsh case. PhD thesis. Berlin, Freie Universität.

TERMCAT, CENTRE DE TERMINOLOGIA (2018): <www.termcat.cat>.

VAn Dongera, R., C. VAN DeR Meer \& R. Sterk (2017): Research for CULT Committee Minority languages and education: best practices and pitfalls. Brussels, European Parliament, Policy Department for Structural and Cohesion Policies.

VILLA, D.J. (2002): «Integrating technology into minority language preservation and teaching efforts: an inside job», en Language Learning \& Technology 6-2, 92-101.

WAGNer, M. (2013): «Luxembourgish on Facebook», en Social Media and Minority Languages. Convergence and the Creative Industries. E. Jones \& E. Uribe-Jongbloed (eds.). Bristol, Multilingual Matters: 87-98.

WORLD BANK GROUP (2018): <www.worldbank.org>.

Xunta Pola Defensa de la Llingua Asturiana (1996): Llibru blancu de la recuperación y normalización llingüística n’Asturies. Uviéu, Xunta Pola Defensa de la Llingua Asturiana. 